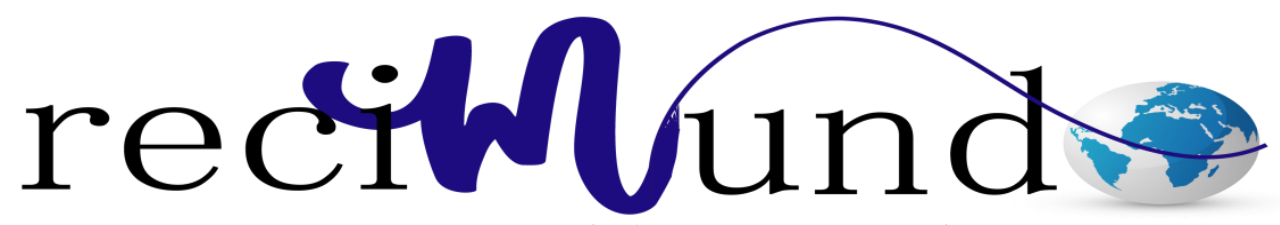

Revista Cientifica Mundo de la Investigación y el Conocimiento

Jessica Leticia Castro Tacuri ${ }^{\text {a }}$; Emma Elizabeth Sacon Martinez ${ }^{\text {b; Azucena }}$ Zeneida Rosado Briones ${ }^{\mathrm{c}}$

Planificar, necesidad para una administración efectiva

Revista Científica Mundo de la Investigación y el Conocimiento. Vol. 2 núm., 1, febrero, ISSN: 2588-073X, 2018, pp. 427-445

DOI: 10.26820/recimundo/2.1.2018.427-445

Editorial Saberes del Conocimiento

Recibido: 05/12/2017

Aceptado: 10/02/2018

a. Ingeniera Comercial; Asistente de Rectorado del Instituto Tecnológico Superior Babahoyo; jessicast2017@gmail.com

b. Ingeniera en Gestión Empresarial.

c. Contador Público Autorizado; Instituto Tecnológico Superior Ciudad de Valencia; azurosbri@gmail.com 


\section{Planificar, necesidad para una administración efectiva}

Vol. 2, núm. 1., (2018)

Jessica Leticia Castro Tacuri; Emma Elizabeth Sacon Martinez; Azucena Zeneida Rosado Briones

\section{RESUMEN}

La administración está presente en cualquier ámbito de la vida, la acción directiva pudiera ser aquella encaminada a lograr de forma creativa, sistémica y humanista la aplicación del proceso administrativo de planificar, organizar, dirigir y controlar, en la intención de lograr una combinación armónica de los recursos técnicos, económicos, financieros y el talento humano de los cuales dispone una organización, lo cual puede ser posible mediante el desarrollo de competencias. Entonces, las ciencias administrativas, como todas las ciencias, desarrollan conocimiento con el propósito de solucionar problemas propios de las funciones asumidas por cada actor de una organización. Nos basamos en la recolección de información disponible en los índices académicos digitales que cumplen con las características descritas para ellos por la sociedad del conocimiento. Tomamos los aportes teóricos de la Administración, revisando sus características, principios y nuevas teorías para resumir, analizar y sintetizar ideas específicas para generar un conjunto de ideas que podemos probar o desechar, de esta manera se genera un conocimiento científico, propósito final de una investigación científica de corte académico. La investigación concluye que los gerentes podrían resistirse a planificar por muchas razones, una de ellas sería debido a los fracasos que ha tenido este proceso a lo largo de la historia, no obstante, se debe seguir planificando porque se obtienen mayores beneficios. En estos casos la planificación siempre sensibiliza el clima laboral lo que compromete por completo cualquier objetivo efectivo de la administración pues no se logra una organización per se, por tanto, el objetivo individual supera merecer la oportunidad de alcanzar objetivos corporativos.

Palabras claves: Administración, planificación, gerencia, ciencia, efectividad. 


\title{
Planificar, necesidad para una administración efectiva
}

Vol. 2, núm. 1., (2018)

Jessica Leticia Castro Tacuri; Emma Elizabeth Sacon Martinez; Azucena Zeneida Rosado

Briones

\begin{abstract}
The administration is present in any area of life, the directive action could be that aimed at creatively, systemically and humanistically achieving the application of the administrative process of planning, organizing, directing and controlling, with the intention of achieving a harmonious combination of the technical, economic, financial and human resources available to an organization, which may be possible through the development of skills. Then, administrative sciences, like all sciences, develop knowledge with the purpose of solving problems inherent to the functions assumed by each actor of an organization. We rely on the collection of information available in digital academic indexes that meet the characteristics described for them by the knowledge society. We take the theoretical contributions of the Administration, reviewing their characteristics, principles and new theories to summarize, analyze and synthesize specific ideas to generate a set of ideas that we can prove or discard, in this way a scientific knowledge is generated, the final purpose of an investigation scientific academic The investigation concludes that managers could resist to plan for many reasons, one of them would be serious due to the failures that this process has had throughout history, nevertheless, it should continue to be planned because greater benefits are obtained. In these cases the planning always sensitizes the work environment which completely compromises any effective objective of the administration because an organization is not achieved per se, therefore, the individual objective exceeds deserving the opportunity to achieve corporate objectives.
\end{abstract}

Keywords: Administration, planning, management, science, effectiveness. 


\section{Planificar, necesidad para una administración efectiva}

Vol. 2, núm. 1., (2018)

Jessica Leticia Castro Tacuri; Emma Elizabeth Sacon Martinez; Azucena Zeneida Rosado Briones

\section{Introducción.}

La administración está presente en cualquier ámbito de la vida, la acción directiva pudiera ser aquella encaminada a lograr de forma creativa, sistémica y humanista la aplicación del proceso administrativo de planificar, organizar, dirigir y controlar, en la intención de lograr una combinación armónica de los recursos técnicos, económicos, financieros y el talento humano de los cuales dispone una organización, lo cual puede ser posible mediante el desarrollo de competencias (Zambrano, 2003). Chiavenato (2002) afirma los más recientes enfoques administrativos destacan que las personas hacen las diferencias en las organizaciones. (Zambrano \& Marval, 2008)

El conocimiento que es todo el conjunto de cogniciones y habilidades con los cuales los individuos suelen solucionar problemas, comprende tanto la teoría como la práctica, las reglas cotidianas al igual que las instrucciones para la acción, el conocimiento se basa en datos e información, pero a diferencia de éstos siempre está ligado a las personas; forma parte integral de los individuos y representa las creencias de éstos acerca de las relaciones causales (Probst, Raub y Romhardt, 2001, p. 24). (Pereira, 2011)

Entonces, las ciencias administrativas, como todas las ciencias, desarrollan conocimiento con el propósito de solucionar problemas propios de las funciones asumidas por cada actor de una organización.

Fayol (1987) trata de obtener una enumeración "todas las operaciones a que las empresas dan lugar" y clasifica sus actividades: 


\section{Planificar, necesidad para una administración efectiva}

Vol. 2, núm. 1., (2018)

Jessica Leticia Castro Tacuri; Emma Elizabeth Sacon Martinez; Azucena Zeneida Rosado

Briones

1. Actividades técnicas: producción, transformación, fabricación.

2. Actividades comerciales: compras, ventas, intercambios.

3. Actividades financieras: captación y administración de capitales.

4. Actividades de seguridad: protección de los bienes de las personas.

5. Actividades contables: inventarios, balances, costos, etc.

6. Actividades administrativas: previsión, organización, dirección, coordinación, control. (Solval, 2015)

Planificar es parte esencial de la administración, sea cual sea el área o sentido del gerente.

Para que se las actividades administrativas cumplan una función efectiva deben aplicarse en ellas algunos principios administrativos que enumeramos:

1. División del trabajo

2. Autoridad y responsabilidad

3. Disciplina

4. Unidad de dirección

5. Subordinación del interés individual al interés general

6. Remuneración 


\section{Planificar, necesidad para una administración efectiva}

Vol. 2, núm. 1., (2018)

Jessica Leticia Castro Tacuri; Emma Elizabeth Sacon Martinez; Azucena Zeneida Rosado Briones

7. Grado de descentralización

8. Jerarquía

9. Orden

10. Equidad

11. Estabilidad del personal

12. Iniciativa

13. Unión del personal (Solval, 2015)

Enfocando este estudio en el ámbito empresarial nos proponemos revisar la bibliografía disponible en cuanto al estudio de la ciencia administrativa que valide la vigencia de la necesidad de planificar para conjugar la acción administrativa en la efectividad de los procedimientos gerenciales.

\section{Materiales y métodos.}

El estudio merece por parte del investigador tener un plan también. Nos basamos en la recolección de información disponible en los índices académicos digitales que cumplen con las características descritas para ellos por la sociedad del conocimiento. Tomamos los aportes teóricos de la Administración, revisando sus características, principios y nuevas teorías para resumir, analizar y sintetizar ideas específicas para generar un nuevo paradigma, con él un conjunto de ideas que podemos probar o desechar, 


\section{Planificar, necesidad para una administración efectiva}

Vol. 2, núm. 1., (2018)

Jessica Leticia Castro Tacuri; Emma Elizabeth Sacon Martinez; Azucena Zeneida Rosado

Briones

de esta manera se genera un conocimiento científico, propósito final de una investigación científica de corte académico.

Para validar lo anterior, considerando la siguiente idea:

En la construcción de cualquier marco teórico hay que precisar que un proceso de investigación no es más que una continuidad de momentos metodológicos ó fases, que son posibles de distinguir a medida que se van moldeando, dentro de un camino de carácter dinámico y procesal (no lineal) que deberá recorrer el investigador; el conocimiento es un organismo en crecimiento, de ahí que cambie y se enriquezca con las nuevas experiencias, de modo tal que pueda pasar de un estado de menor conocimiento (inicial) a un estado más complejo y más eficaz (final), donde el trabajo de cada pesquisa o exploración en particular es parte de un esfuerzo continuo y mucho mayor en el desarrollo de nuevos hallazgos. Puledda (1996) menciona que para Marx "el trabajo no es solamente una actividad práctica, sino también una categoría del conocimiento y es lo que constituye justamente la vida y la conciencia de la especie" (Di Marco, 2015)

Precisamente, es parte de la investigación considerar la experiencia del autor en el ámbito del desarrollo de las capacidades profesionales considerar la variable en estudio, pues es la planificación la fase que principalmente falla a la hora de evaluar la efectividad de la aplicación de la ciencia administrativa. Es debido a ello que se considera indispensable volver a las bases teóricas para identificar los elementos validos rescatables para potenciar la gerencia en cualquier área de aplicación posible. 


\section{Planificar, necesidad para una administración efectiva}

Vol. 2, núm. 1., (2018)

Jessica Leticia Castro Tacuri; Emma Elizabeth Sacon Martinez; Azucena Zeneida Rosado Briones

\section{Resultados.}

Las funciones Administrativas en un enfoque sistemático conforman el proceso administrativo, cuando se consideran aisladamente los elementos Planificación, Organización, Dirección y Control, son solo funciones administrativas, cuando se consideran estos cuatro elementos en un enfoque global de interacción.

Dado que las organizaciones existen por un objetivo concreto, entonces alguien debe definir ese objetivo y los medios para cumplirlo. Ese alguien es el gerente. Planificar abarca la definición de las metas de la organización, el establecimiento de una estrategia general para alcanzar esas metas y el desarrollo de una jerarquía minuciosa de los planes para integrar y coordinar las actividades. Establecer metas sirve para no perder de vista el trabajo que se hará y para que los miembros de la organización fijen su atención en las cosas importantes. (Robbins \& Decenzo, 2002)

Resulta común en el contexto organizacional, la expresión “el problema es gerencial”. Es decir, la multiplicidad de problemas cuya explicación se asocia a la administración y/o gerencia se endosan a su actor principal, indistintamente de la denominación recibida: presidente, director, gerente, coordinador, y a la forma como éste ejecuta las funciones del proceso administrativo. Lo anterior implica superar esquemas tradicionales, no solo relativas a sus funciones básicas: docencia, investigación y extensión o a cambios radicales para la producción y/o aprehensión del conocimiento, sino a la adopción de procesos administrativos tendentes a desarrollarlos en el marco de suficiencia en las competencias del talento humano, implica pues, el ejercicio de una acción directiva plena, integral que viabilice conjugar en la práctica pensamiento y acción. 


\section{Planificar, necesidad para una administración efectiva}

Vol. 2, núm. 1., (2018)

Jessica Leticia Castro Tacuri; Emma Elizabeth Sacon Martinez; Azucena Zeneida Rosado

Briones

Integrar ambos elementos, para la transformación de procesos inherentes a lo administrativo en el ámbito universitario exige, formación en competencias, vistas no de forma acrítica o instrumental tras la búsqueda de la eficacia o eficiencia administrativa. Esto ratifica la importancia de las competencias en el talento humano para lograr el desarrollo de procesos administrativos alejados de la racionalidad tradicional que mediatiza la acción directiva y la circunscribe a un conjunto de técnicas y procedimientos en buena proporción desprovista de la visión humanista, integral, holística, necesarias para direccionar, dada la complejidad del mundo moderno. (Zambrano \& Marval, 2008)

\section{La Planeación}

Fija tiempos de las determinaciones de las unidades que se plantean los objetivos. La determinación de los objetivos y elección de los cursos de acción para lograrlos, con base en la investigación y elaboración de un esquema detallado que habrá de realizarse en un futuro.

\section{Principios de la Planeación}

Los principios de la administración son verdades fundamentales de aplicación general que sirven como guías de conducta a observarse en la acción administrativa.

- Factibilidad: Lo que se planea ha de ser realizable.

- Objetividad y cuantificación: establece la necesidad de utilizar datos objetivos tales como estadística, estudios de mercado, cálculos probabilísticos, modelos matemáticos y datos numéricos, al elaborar planes para reducir al mínimo los riesgos. 


\section{Planificar, necesidad para una administración efectiva}

Vol. 2, núm. 1., (2018)

Jessica Leticia Castro Tacuri; Emma Elizabeth Sacon Martinez; Azucena Zeneida Rosado Briones

- Flexibilidad: es conveniente establecer márgenes de holgura que permitan afrontar situaciones imprevistas.

- Unidad: todos los planes específicos de la empresa deben integrarse a un plan general y dirigirse al logro de los propósitos y objetivos generales.

- Intercambio de estrategias: cuando un plan se extiende en relación al tiempo (largo plazo), será necesario rehacerlo completamente.

- Los planes, en cuanto al periodo establecido para su realización se pueden clasificar en:

o Corto Plazo: menor o igual a un año. Estos a su vez pueden ser: Inmediatos: hasta seis meses. Mediatos: mayor de seis o menor de doce meses.

- Mediano Plazo: de uno a tres años.

○ Largo Plazo: mayor a tres años.

\section{Etapas de la Planeación}

Premisas: Las premisas son suposiciones que se deben considerar ante aquellas circunstancias o condiciones futuras que afectaran al curso en que va a desarrollarse el plan. Las premisas de acuerdo a su naturaleza pueden ser:

1. Internas: cuando se originan dentro de la empresa y pueden influir en el logro de los propósitos.

2. Externas: son factores o condiciones cuyo origen es ajeno a la empresa, pero pueden tener efecto decisivo en el desarrollo de sus actividades. Están premisas externas pueden ser: 


\section{Planificar, necesidad para una administración efectiva}

Vol. 2, núm. 1., (2018)

Jessica Leticia Castro Tacuri; Emma Elizabeth Sacon Martinez; Azucena Zeneida Rosado

Briones

a. De carácter político.

b. De carácter legal.

c. Económicas.

d. Sociales.

e. Técnicas.

f. Otros factores.

Investigación: La investigación es un proceso que, mediante la aplicación del método científico, procura obtener información relevante y fidedigna con el fin de explicar, describir y predecir la conducta de los fenómenos. La investigación es trascendental en la planeación, ya que proporciona información a las etapas de la misma para que se lleve a cabo racionalmente.

Reglas: Dentro del procedimiento tenemos las reglas; las cuales son aplicables al "procedimiento" y al "método"; las reglas de por tanto, son normas que se refieren a una acción específica y definida; o sea, que una regla dice con exactitud qué debe hacerse y que no debe hacerse. -Las regla no deja campo o decisión o elección al jefe en quien se delega autoridad, sino que tan solo le permite analizar si ella es aplicable al caso concreto de que se trate.

Políticas. Las políticas son guías para orientar la acción, son criterios, lineamientos generales a observar en la toma de decisiones, sobre problemas que se repiten una y otra vez dentro de una organización. 


\section{Planificar, necesidad para una administración efectiva}

Vol. 2, núm. 1., (2018)

Jessica Leticia Castro Tacuri; Emma Elizabeth Sacon Martinez; Azucena Zeneida Rosado Briones

Objetivos. Representan los resultados que la empresa espera obtener, son fines para alcanzar, establecidos cuantitativamente y determinados para realizarse transcurrido un tiempo específico.

\section{Clasificación de los objetivos:}

1. Estratégicos o generales: comprenden toda la empresa y se establecen a largo plazo.

2. Tácticos o departamentales: se refieren a un área o departamento de la empresa, se establecen a corto o mediano plazo

3. Operacionales o específicos se establecen en niveles o secciones más especificas de la empresa e invariablemente son a corto plazo.

Presupuestos: Considerado como un plan, un presupuesto es la expresión de los resultados esperados en términos numéricos; hay varios tipos de presupuesto proyectado de ganancias y pérdidas. Hacer un presupuesto es planear, el presupuesto es un instrumento de control que permite consolidar los planes de una empresa. Además de su uso en la planeación, el presupuesto también se utiliza para el control y la coordinación. Como fuente de coordinación, el presupuesto proporciona información respecto a los planes que se han hecho, asegurándose de que estén en equilibrio con cada uno de los otros planes.

Diferentes tipos de presupuestos:

Específicos: Comúnmente se usa en el gobierno, consiste esencialmente en que establece una suma de dinero como límite a gastar o a invertir en una determinada actividad o programa. 


\section{Planificar, necesidad para una administración efectiva}

Vol. 2, núm. 1., (2018)

Jessica Leticia Castro Tacuri; Emma Elizabeth Sacon Martinez; Azucena Zeneida Rosado

Briones

Fijos: Implican un plan que no cambia, aunque cambien sus ventas o la producción; el estándar o unidad de medida no cambia.

Flexibles o variables: Permite revisar los gastos y los costos de producción; es más fácil de utilizar en el control de operaciones que el presupuesto específico. Existe además otro tipo de clasificación como la siguiente:

a) Presupuesto de operación: incluye la planeación de las operaciones para el siguiente período.

b) Presupuesto de capital: demuestra la planeación de los cambios en los activos fijos; y

c) Presupuesto financiero: establece el origen y aplicación de fondos, trata de los movimientos de efectivo.

Programas: Los programas son los planes mismos; pero en los cuales no solo se fijan los objetivos y la secuencia de operaciones, sino principalmente se hace referencia al tiempo requerido para realizar cada de una de sus partes. -Es la consecuencia cronológica que confiere vitalidad y sentido práctico a un plan". Los programas pueden ser a corto plazo generalmente a un año y de largo plazo cuando excede ese período. Los programas tienen como fundamento el capital necesario y los presupuestos operacionales; los programas están constituidos por metas, políticas, procedimientos, reglas, asignación de áreas, recursos a emplearse y otros elementos necesarios para llevar a cabo un curso de acción.

Estrategias: Las estrategias son cursos de acción general o alternativas, que muestran la dirección y el empleo general de los recursos y esfuerzos para lograr los objetivos en las 


\section{Planificar, necesidad para una administración efectiva}

Vol. 2, núm. 1., (2018)

Jessica Leticia Castro Tacuri; Emma Elizabeth Sacon Martinez; Azucena Zeneida Rosado Briones

condiciones más ventajosas. Las estrategias en la empresa nacen como una respuesta para afrontar los retos que implican la competencia, y la vida de la empresa en sí.

Al establecer estrategias es conveniente seguir tres etapas:

1. Determinación de los cursos o alternativas: consiste en buscar el mayor número de alternativas para lograr cada uno de los objetivos.

2. Evaluación: analizar y evaluar cada una de las alternativas tomando en consideración las ventajas y desventajas de cada una de ellas.

3. Selección de alternativas: considerar las alternativas más idóneas en cuanto a factibilidad y ventajas. Para que las estrategias sean operables y cumplan con su función, es necesario:

- Asegurarse de que sean consistentes y contribuyan al logro del objetivo.

- Determinarlas con claridad.

- No confundir las estrategias con las tácticas, ya que estas últimas combinan la acción con los medios para alcanzar el objetivo.

- Considerar las experiencias pasadas para su establecimiento; esto permitirá sugerir un mayor número de cursos de acción.

- Analizar las consecuencias que pudieran presentarse al momento de aplicarlas.

- Al establecerlas, auxiliarse de técnicas de investigación y de planeación.

Procedimientos: Detallan la forma exacta en que debe desarrollarse una actividad; y son guías de acción más que de pensamiento. Los procedimientos son aquellos planes formulados 


\section{Planificar, necesidad para una administración efectiva}

Vol. 2, núm. 1., (2018)

Jessica Leticia Castro Tacuri; Emma Elizabeth Sacon Martinez; Azucena Zeneida Rosado

Briones

dentro de las políticas establecidas, cuya finalidad es la de señalar la secuencia cronológica más eficiente, destinada a obtener los mejores resultados en cada función concreta realizada dentro de una empresa o grupo social. Los procedimientos son más definidos que las políticas y se aplican a actividades específicas para la realización de ciertas metas bien definidas. Los procedimientos se dan en todos los niveles de una empresa, pero son más frecuentes en los niveles de operación, a diferencia de las políticas que se dan en los niveles más altos. Un procedimiento es la descripción de cómo cada una de las serie de tareas se van a llevar a cabo, cuando va a realizarse y por quién, como por ejemplo: Un conjunto de instrucciones específicas para procesar órdenes.

Métodos: Es una descripción de cómo un paso del procedimiento puede llevarse a cabo de la mejor forma posible, ejemplo: la técnica específica en la aplicación de un test de aptitudes es un método; mientras que la secuencia de pasos para emplear personal constituye un procedimiento.-La simplificación del trabajo (eliminación de los movimientos innecesarios para hacer un trabajo) debe comenzar con el estudio de los procedimientos con el empleo de métodos adecuados un gerente podrá lograr mayor eficiencia, comparar fácilmente los rendimientos, elaborar bases para determinar la capacidad efectiva del personal, lograr calidad uniforme de productos o servicios, etc.

Propósitos: Las aspiraciones fundamentales o finalidades de tipo cualitativo que persigue en forma permanente o semipermanente a un grupo social.

Las siguientes cuatro características los diferencian de los objetivos.

a. Son básicos o trascendentales, porque constituyen el fundamento de los demás elementos. 


\section{Planificar, necesidad para una administración efectiva}

Vol. 2, núm. 1., (2018)

Jessica Leticia Castro Tacuri; Emma Elizabeth Sacon Martinez; Azucena Zeneida Rosado Briones

b. Genéricos o cualitativos, porque no se expresan en términos numéricos.

c. Permanentes, porque permanecen vigentes durante el periodo de vida de la organización.

d. Semipermanentes, porque pueden abarcar un periodo determinado (Solval, 2015)

\section{Conclusiones.}

La administración científica surge para agilizar, racionalizar y aumentar la productividad. La administración en el mundo moderno es indispensable, no solamente en las empresas e industrias sino en todo tipo de organizaciones. El papel que desempeña la administración entre las organizaciones productivas es sumamente importante, ya que las complejas relaciones que se producen en las industrias modernas hacen necesario organizar racionalmente el trabajo, entonces solamente a través de técnicas y procesos administrativos es que se puede cumplir con eficacia los procesos productivos, distributivos y de consumo de bienes y servicios que las empresas producen. En los organismos grandes la administración es indiscutible y esencial porque no podrán actuar si no fuera a base de una administración sumamente técnica, en cambio para las medianas y pequeñas empresas la ciencia administrativa ofrece la única oportunidad de competir con otras porque garantiza una mejor coordinación de los elementos con los que cuenta como clasificación de la mano de obra, maquinarias, tecnologías etc. (Hurtado, 20018)

Planificar es parte de los 4 elementos del proceso administrativo, es importate que los gerentes tengan la capacidad de entender que estos procesos necesariamente se desarrollan simultáneamente pues se relacionan entre sí y repercuten dinámicamente en los otros. No hay una efectividad en la administración si la planificación, la organización, la dirección o el control 


\section{Planificar, necesidad para una administración efectiva}

Vol. 2, núm. 1., (2018)

Jessica Leticia Castro Tacuri; Emma Elizabeth Sacon Martinez; Azucena Zeneida Rosado

Briones

fallan, mas es en este orden que se puede diseñar una administración correcta garantizando la efectividad y la eficacia a través de la aplicación y evaluación de los controles diseñados

El Proceso Administrativo tiene dos fases que se denominan Mecánica, Dinámica, la Previsión es parte de la fase Mecánica, en ella podemos examinar el futuro, fijar los objetivos y misiones de la empresa.

El proceso administrativo, desde el aporte al desarrollo de la ciencia administrativa admite ahora 6 elementos los cuáles son la planeación, organización, previsión, dirección, control, integración. En conde la previsión y la integración son dos elementos adheridos a los 4 iniciales propuestos en la teoría desarrollada por Fayol (1987)

El proceso administrativo es una técnica que podemos aplicar dentro de una empresa para un buen desarrollo empresarial, la planeación es un proceso por el cual podemos medir el tiempo para determinar las actividades que se van a desarrollar. (Solval, 2015)

La dirección es el elemento del proceso administrativo responsable de coordinar el talento humano en las empresas. Es fundamental, pues su racionalidad viabiliza alcanzar lo planificado, dada la autoridad y la coordinación de actividades que permite. Las habilidades humanas presentes en la dirección, comunicación, liderazgo, motivación, toma de decisiones y, en el nuevo contexto, la integración, la ratifican como el corazón del proceso administrativo. El carácter dinámico de dicha función o proceso permite asumir el modelo humanista, en contraposición al mecanicista cuyos postulados aún se intentan superar y con el cual se identifican en mayor proporción las restantes etapas del proceso administrativo. La dirección reviste un carácter eminentemente humano, exige para la solución de problemas integrar el 


\section{Planificar, necesidad para una administración efectiva}

Vol. 2, núm. 1., (2018)

Jessica Leticia Castro Tacuri; Emma Elizabeth Sacon Martinez; Azucena Zeneida Rosado Briones

tiempo y las circunstancias, es necesario interactuar día a día, minuto a minuto, no sólo a partir de políticas, planes, programas y procedimientos, sino además debe regirse por valores, premisas y principios que en todo caso guían la acción directiva en una perspectiva humanista. Al respecto, Ballina (2000:91) explica "toda dirección está influenciada por la época en la cual opera”. (Zambrano \& Marval, 2008)

La gestión de organizaciones tiende a ser muy afectada por las características de la cultura nacional y organizacional donde ésta se desarrolla. Esto último es particularmente cierto para los aspectos de gestión relacionados con la administración de personas, como lo son los estilos de gestión. La cultura de trabajo se puede entender como el conjunto de valores y normas de comportamiento que caracterizan el ambiente laboral de una organización (Hofstede, 1991). Estos valores y normas de comportamiento son usados como reglas para tomar decisiones y definen las relaciones entre las personas y los grupos al interior de la organización. El impacto de la cultura local afecta la implementación de prácticas de gestión. Es importante entender las características de la cultura laboral de un país para poder comprender cuáles formas de trabajo y de relación son más aceptadas y/o promovidas por la cultura y cuáles pueden ser consideradas contraculturales (Tannenbaum y Davis, 1969), y más difíciles de asimilar. (Raineri, 2006)

Los gerentes podrían resistirse a planificar por muchas razones, una de ellas seria debido a los fracasos que ha tenido este proceso a lo largo de la historia, no obstante la investigación refiere a que se debe seguir planificando pese a los fracaso porque se obtienen mayores beneficios cuando las organizaciones se planifican. El reto gerencial se enfoca en lograr superar la resistencia de los gerentes de niveles medios o bajos que no quieren imposición de planes y procesos de planificación que vienen de los niveles gerenciales superiores puesto que esto va en 


\section{Planificar, necesidad para una administración efectiva}

Vol. 2, núm. 1., (2018)

Jessica Leticia Castro Tacuri; Emma Elizabeth Sacon Martinez; Azucena Zeneida Rosado

Briones

contra de su propia flexibilidad y su madurez en el campo laboral como también asumir, cuando la planificación se hace desde niveles mas bajos de gerencia e impone el plan hacia las jerarquías superiores. (Mintzberg, Bryan, \& Voyer, 1997)

En estos casos la planificación siempre sensibiliza el clima laboral lo que compromete por completo cualquier objetivo efectivo de la administración pues no se logra una organización per se, por tanto el objetivo individual supera merecer la oportunidad de alcanzar objetivos corporativos.

\section{Bibliografía.}

Di Marco, R. (2015). En busca del origen del conocimiento: El dilema de la realidad. Praxis, II, $150-162$.

Hurtado, D. (2018). Principios de la Administración. Medellin: Fondo Editorial ITM.

Mintzberg, H., Bryan, J., \& Voyer, J. (1997). El proceso estratégico: conceptos, contextos y casos. Naucalpan de Juárez: Pretice Hall Hispanoamericana, S.A.

Pereira, H. (2011). Implementación de la Gestión del Conocimiento en la empresa. Éxito Empresarial. $\quad 135, \quad 1-6 . \quad$ Obtenido de http://www.cegesti.org/exitoempresarial/publicaciones/publicacion_135_310111_es.pdf

Raineri, A. (2006). Estilos de dirección como determinantes del clima laboral en Chile. Revista ABANTE,9(1) , 3-33.

Robbins, S., \& Decenzo, D. (2002). Fundamentos de la Administración. 3ra Edición. México: Pearson Educación.

Solval, J. (22 de 03 de 2015). El proceso Administrativo. Obtenido de http://www.academia.edu/download/36395567/El_Proceso_AdministrativoIII_-_SEjwf.pdf.

Zambrano, B., \& Marval, E. (2008). Acción directiva: Un enfoque centrado en competencias . Revista venezolana de gerencia. 12 (44), 616-636. 